\title{
Shooting time's arrow
}

\section{The laws of physics mean that the Universe is slowly dying.}

\section{The Fabric of the Cosmos: Space, Time and the Texture of Reality \\ by Brian Greene \\ Alfred Knopf: 2004. 512 pp. \$28.95 \\ Allen Lane: 2004. $£ 25$ \\ Paul Davies}

The most apocalyptic pronouncement in the history of science was made by the physicist Hermann von Helmholtz in 1854. The Universe, von Helmholtz declared, is dying. His pessimistic prediction was based on the second law of thermodynamics, according to which the entire cosmos is on a one-way slide towards a state of complete disorder, or maximum entropy, from which it will be unable to extricate itself.

The basis of the dying Universe is easy to explain. We are surrounded by processes that have a definite directionality to them: people grow old, cars rust, cliffs are eroded, sandcastles get washed away. Take a movie of any familiar scene and run it backwards - people will laugh, because the world in reverse looks so preposterous.

The same directionality pervades the cosmos. The Sun, for example, is not the immutable orb it may seem. Over billions of years it has consumed much of its fuel, and eventually it will burn out and die. The same goes for all stars. Irreversible decay and degeneration spell universal heat death, just as von Helmholtz proclaimed.

But this incontestable fact conceals a deep mystery. The world about us may display a conspicuous 'arrow of time', but the underlying laws of physics are, with a minor exception, completely symmetric. At the level of individual atoms, there is nothing to distinguish future from past. So somehow, between atom and cosmos, the temporal lopsidedness of the Universe emerges. How this happens is one of the longest-running debates in science.

I became fascinated by this mystery after hearing a lecture delivered at the Royal Society in London by Fred Hoyle in 1968, and I have returned to it again and again throughout my career. Early on it became obvious to me that the subject was marred by widespread misconceptions and muddled thinking, even among some of the giants of theoretical physics. It is therefore extremely heartening that Brian Greene has written such a sensible and consistent account of this tantalizing topic, where so many other authors have merely added to the confusion.

Following the success of his book on the unification of physics, The Elegant Universe, Greene has turned his attention to the nature of space and time. Before zeroing in on the

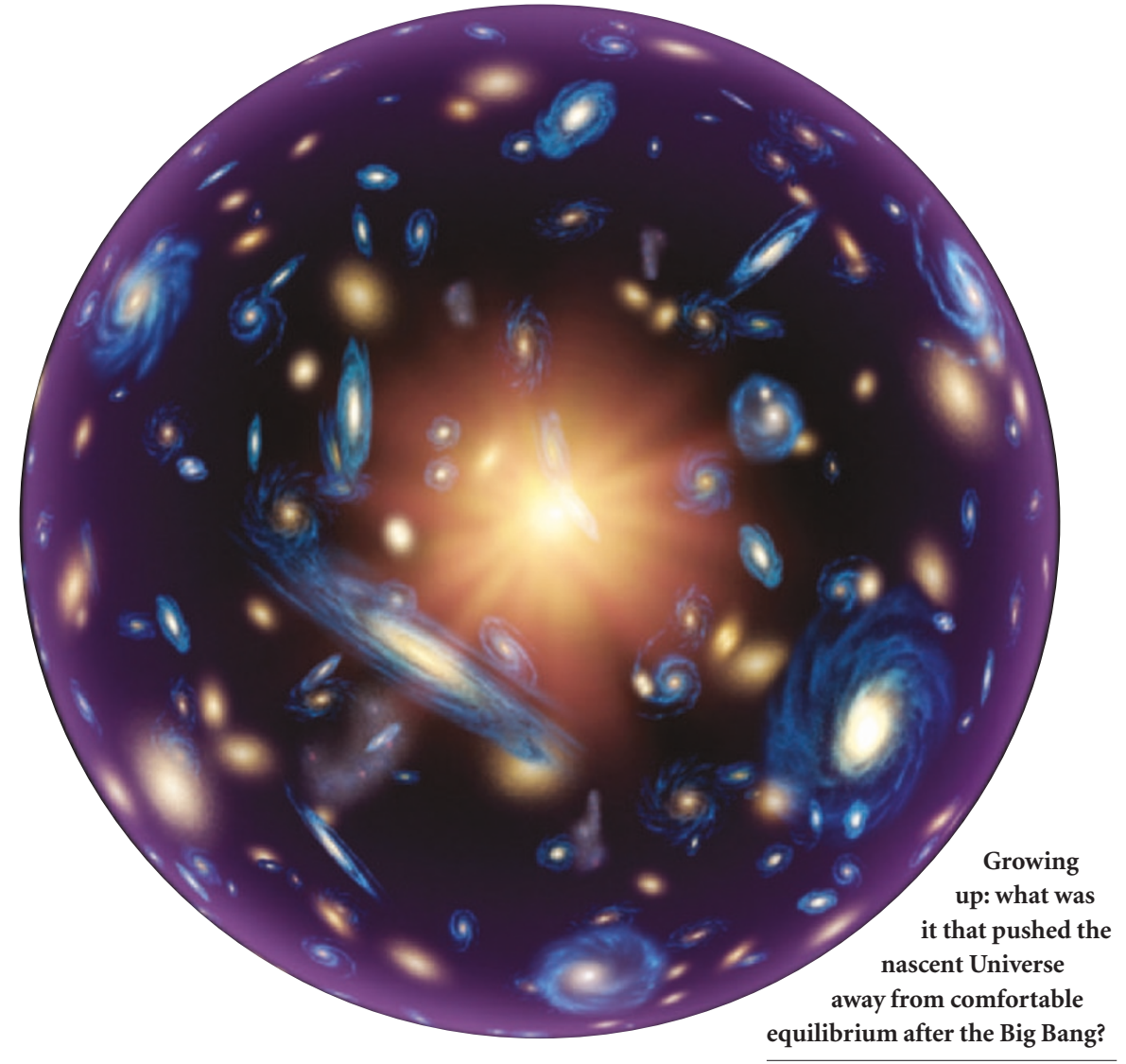

vexed issue of time's elusive arrow, he covers some familiar territory: the theory of relativity, basic cosmology, Mach's principle, quantum non-locality and the Bell inequalities, Wheeler's delayed-choice experiment and the many-universes interpretation of quantum mechanics. Greene even tackles, but does not resolve, the vexed issue of the flow of time - that most familiar of human experiences, which is bafflingly absent from physicists' descriptions of the world.

One way to think of the cosmic arrow of time is to regard the Universe as a gigantic clock slowly running down. The problem is to explain how it was wound up in the first place. Cosmologists naturally look to the Big Bang as the start of the whole show. But this immediately presents a puzzle. We have available a snapshot of the Universe shortly after its birth, in the form of a thermal map of the sky taken from satellites such as the Wilkinson Microwave Anisotropy Probe and various ground-based instruments. The data show the fading afterglow of the Big Bang, and enable cosmologists to reconstruct the details of its primordial thermodynamic state. The result is striking: just 380,000 years after its birth, the Universe looked virtually dead already, consisting of uniform hot gas spread through space in a state extremely close to the heat death envisaged by von Helmholtz. So, if the young Universe was close to thermodynamic equilibrium then, how has it come alive again and achieved such a low-entropy state today?

Greene correctly identifies the solution of this puzzle. The key lies with gravity. This universal force seizes any tiny irregularity in the distribution of matter and amplifies it. Over billions of years, the smooth hot gases of the early Universe have become clumpy assemblages of galaxies, stars and black holes. This trend from smooth to clumpy constitutes gravity's very own arrow of time and trumps the rise in the entropy of matter. The upshot is that a Universe that starts out smooth, with matter in thermodynamic equilibrium, can end up clumpy, with matter dragged far from equilibrium. Since the time of the Big Bang, the entropy of matter has risen, but the maximum possible entropy has of the Universe. Our very existence depends on the entropy gap that this has created.

The quest for the source of time's arrow can therefore be traced back, somewhat tortuously, to the fact that the Universe began in a relatively smooth state. When I worked on this problem in the 1970s, the initial smoothness had to be simply accepted as an unexrisen even faster, mostly due to the expansion 
plained initial condition. Today, however, there is a ready explanation in the form of the 'inflationary Universe' scenario, involving a burst of frenetic expansion shortly after the cosmic origin. Any pre-existing irregularities would have been smoothed away by this abrupt and huge swelling of space.

This pleasing conclusion immediately begs the question of what preceded inflation and why it happened at all — which provides a convenient jumping-off point for Greene to embark on a discussion of the physics of unification. This is familiar territory for him, as he is first and foremost a string theorist. He treats the reader to a review of the current state of string/M theory, with digressions into a bewildering array of topics including higher dimensions, brane worlds, the holographic paradigm, teleportation, wormholes and time travel. If I have a criticism of this book, it is that it packs in so many challenging and abstract topics that it can leave the reader's mind reeling. Sensibly, Greene repeatedly cautions that most of the ideas he gallops across are extremely speculative and are unlikely to be tested experimentally any time soon.

The nature of space and time has exercised the minds of the world's greatest scientists and philosophers for centuries. Mostly they have regarded space and time as simply there - a given. Modern physics has shown that they are, in fact, things, just as particles of matter are things. The heady developments described in this book hold out the promise that we may one day explain how space and time have come to exist, and why they possess the properties that they do. Paul Davies is at the Australian Centre for Astrobiology, Macquarie University, Sydney, New South Wales 2109, Australia. His latest book is How to Build a Time Machine.

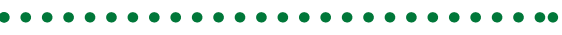 Touring artificial minds}

\section{Alan Turing: Life and Legacy of a Great Thinker \\ edited by Christof Teuscher \\ Springer: 2004. 542pp.£46, \$69.95, €59.95}

\section{John L. Casti}

In 1999, Time magazine made Albert Einstein its 'man of the century' for the work that changed our view of time and space. It's difficult to argue too strenuously with this choice. But when it comes to scientists who have affected daily life, a far better choice would be Alan Turing, the British mathematician turned computer scientist whose invention in 1936 of what is now called the Turing machine was the theoretical backbone for every one of the zillions of computers in use today. Not only did Tur-

ing provide the key theoretical element for computing machines, he helped to build one of the first electronic computers, in Manchester, UK, shortly after the Second World War.

This book is the outgrowth of a workshop held in Lausanne, Switzerland, in June 2002 to honour the ninetieth anniversary of Turing's birth on 23 June 1912. Turing's work was so broad and deep that another gathering this year, to mark the fiftieth anniversary of his death, would not be out of place.

It is difficult to find the Machine architect: Alan Turing superlatives to describe

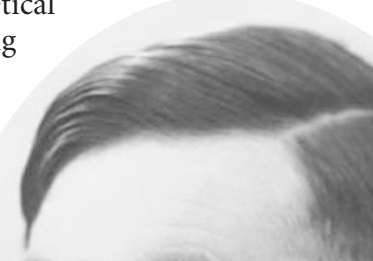

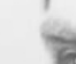
the wonderful job the contributors to this book have done. Every chapter is written in an expository fashion, demanding very little in the way of background knowledge from any scientifically minded reader. The range of topics is also impressive, with sections on Turing's life and thoughts, the theory of computation and the Turing machine, artificial intelligence and the Turing test, the wartime Enigma code-breaking work and, finally, forgotten ideas.

Each section contains between two and seven chapters that explore themes ranging from what Turing might have thought about today's work in 'hypercomputation' - a field that explores information processing beyond the abilities of Turing machines to his ideas on thinking machines and robots. The book's contributors are as sterling a collection of computer scientists, philosophers, engineers and historians as one could ever wish for, including logician Martin Davis, philosophers Daniel Dennett and Jack Copeland, technologist Ray Kurzweil and historian Andrew Hodges.

In her extremely entertaining chapter "Alan's Apple: Hacking the Turing Test", the Italian writer and theatre director Valeria Patera creates a theatrical setting in which eminent figures in artificial intelligence meet in a virtual plane to consider Turing's ideas on thinking machines. A staging of this might be more interesting, intellectually at least, than the rather dull play Breaking the Code that ran so successfully in London and New York some years back.

Two of the more provocative contributions come from Davis, who argues against the ideas put forth by a number of researchers for transcending the Turing barrier in computation, and from Kurzweil, who explains in detail his well-known arguments for why technological progress will occur at such a pace that machine intelligence will surpass the human variety within a few decades.

On the principle that no book is perfect, I have to admit to one small quibble. Given Turing's greatinterest in biological processes, especially near the end of his life, and his pioneering work on what we now call mathematical biology, I was disappointed to see only one of the 20 chapters devoted to that aspect of his work. Of course, no book can do everything, and this short-changing of biology in favour of computing is more of an opportunity than a problem. Nevertheless, a couple more chapters on morphogenesis, artificial life and so on would have really made this book the definitive volume on Turing's work and its implications.

I unreservedly recommend this book to anyone even slightly interested in the continuing role of Turing's work in the development of computer science in particular, and ideas in general. Conference proceedings rarely make for good reading and are generally strange beasts to review. This volume is the exception that proves that rule.

John L. Casti is at Complexica, Santa Fe, New Mexico 87505, USA, and the Institute for Monetary Economics, Vienna, Austria.

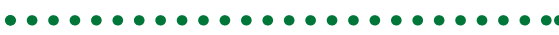 In Newton's long shadow}

\section{From Newton to Hawking: A History of Cambridge University's Lucasian Professors of Mathematics \\ edited by Kevin Knox \& Richard Noakes Cambridge University Press: 2003. 512 pp. $£ 27.50, \$ 45$}

\section{Lewis Pyenson}

For more than 300 years, Cambridge University's Lucasian professors have promoted the mathematical elaboration of nature's laws. The founder of the chair, Henry Lucas, who was awarded an honorary MA by the university in 1636 and who represented Cambridge in both the Short and the Long Parliaments, filled a gap in his university by endowing a professorship in mathematics, 